\title{
Ações de enfermagem para a desospitalização de crianças em ventilação mecânica
}

Nursing actions for the dehospitalization of children under mechanical ventilation Acciones de enfermería para la externación de niños con ventilación mecánica

Larissa de Oliveira Ulisses ${ }^{1}$ id https://orcid.org/0000-0002-8762-2392

Thaysy Andrade Silva Bispo ${ }^{1}$ id https://orcid.org/0000-0003-0253-381

Agatha Barbosa Caldas ${ }^{1}$ id https://orcid.org/0000-0001-8681-7238

Climene Laura de Camargo ${ }^{1}$ iD https://orcid.org/0000-0002-4880-3916 Márcia Maria Carneiro Oliveira ${ }^{1}$ id https://orcid.org/0000-0003-2294-0872

Evaldo Almeida da Silva ${ }^{1}$ id https://orcid.org/0000-0002-1229-4953

Nadirlene Pereira Gomes ${ }^{1}$ iD https://orcid.org/0000-0002-6043-3997

Maria Carolina Ortiz Whitaker ${ }^{1}$ id https://orcid.org/0000-0003-0253-3831

Ulisses LO, Bispo TA, Caldas AB citar: CL, Oliveira MM, Silva EA, et al. Ações de enfermagem para a desospitalização de crianças em ventilação mecânica. Acta Paul Enferm. 2021;34:eAPE000785.

DOI

http://dx.doi.org/10.37689/actaape/2021A0000785

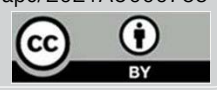

Descritores

Doença crônica; Respiração artificial; Alta do paciente; Criança; Cuidadores

Keywords

Chronic disease; Respiration, artificial; Patient discharge; Child; Caregivers

Descriptores

Enfermedad crónica; Respiración artificial; Alta del paciente; Cuidadores

\section{Submetido}

9 de Abril de 2020

Aceito

1 de Março de 2021

Autor correspondente

Maria Carolina Ortiz Whitaker Email: maria.ortiz@ufba.br

\section{Resumo}

Objetivo: Apreender as ações de enfermagem para a desospitalização de crianças dependentes de ventilação mecânica.

Métodos: Estudo qualitativo, com 15 profissionais de enfermagem de uma unidade de referência nesse cuidado de saúde. Os dados foram obtidos por entrevistas, submetidas à análise de conteúdo e interpretados pela fundamentação do Modelo de Adaptação de Roy.

Resultados: As ações ocorreram pelo levantamento dos problemas, pelo planejamento do cuidado e na avaliação do processo de adaptação para o cuidado após alta hospitalar de crianças com respiração por ventilação mecânica. Essas atividades foram permeadas pela valorização das necessidades individuais, pela observação dos comportamentos e estímulos vivenciados ao longo do preparo para o cuidado domiciliar.

Conclusão: As ações se concentraram no apoio e capacitação dos cuidadores para atender as necessidades das crianças e revelaram a importância da fundamentação teórica de enfermagem para fortalecer e aprimorar cientificamente as ações realizadas.

\section{Abstract}

Objective: To understand nursing actions for the dehospitalization of children dependent on mechanical ventilation.

Methods: This is a qualitative study, with 15 nursing professionals from a reference unit in this health care. The data were obtained through interviews, submitted to content analysis and interpreted by the foundation of Roy's Adaptation Model.

Results: The actions took place by surveying the problems, planning care and assessing the adaptation process for care after hospital discharge of children with mechanical ventilation breathing. These activities were permeated by valuing individual needs, observing the behaviors and stimuli experienced during the preparation for home care.

Conclusion: The actions focused on the support and training of caregivers to meet children's needs and revealed the importance of the theoretical foundation of nursing to strengthen and scientifically improve the actions carried out.

\section{Resumen}

Objetivo: Comprender las acciones de enfermería para la externación de niños dependientes de ventilación mecánica. 
Métodos: Estudio cualitativo con 15 profesionales de enfermería de una unidad de referencia en este tipo de cuidado de la salud. Los datos se obtuvieron mediante entrevistas, que fueron sometidas al análisis de contenido e interpretadas mediante la fundamentación del modelo de adaptación de Roy.

Resultados: Las acciones se realizaron a través de la recopilación de los problemas, la planificación del cuidado y la evaluación del proceso de adaptación para el cuidado luego del alta hospitalaria de niños con respiración por ventilación mecánica. En estas actividades estuvo presente la valorización de las necesidades individuales, la observación de los comportamientos y estímulos vividos a lo largo de la preparación para el cuidado domiciliario.

Conclusión: Las acciones se concentraron en el apoyo y la capacitación de los cuidadores para atender las necesidades de los niños y revelaron la importancia de la fundamentación teórica de enfermería para fortalecer y perfeccionar científicamente las acciones realizadas.

\section{Introdução}

É crescente o número de crianças em condiçẫo crônica de saúde que vivem com dependência de ventilação mecânica, demandando capacitação e preparo no planejamento para as açóes de cuidado. ${ }^{(1,2)}$ Nos Estados Unidos, uma em cada cinco crianças apresentam alguma necessidade especial de saúde. ${ }^{(3)} \mathrm{Na}$ Alemanha, estima-se que 2000 crianças vivem com dependência de ventilação mecânica. ${ }^{(4)}$ No último censo demográfico brasileiro, cerca de $24,1 \%$ da população com idade inferior a 14 anos, apresentavam com algum tipo de deficiência. ${ }^{(5)}$ Embora seja um dado relevante, é importante destacar que os dados epidemiológicos referentes a essa população encontram-se partilhados, conforme comprometimento dos sistemas orgânicos, padronizados pela Classificação Internacional de Doenças (CID), prevendo diferentes graus e tipos de dependências tecnológicas por doenças multissistêmicas, o que dificulta o reconhecimento dessas crianças no cenário nacional. ${ }^{(6)}$

Os avanços tecnológicos e as atuaçóes multiprofissionais, tem favorecido a qualidade de vida dessas crianças com necessidades especiais de saúde (CRIANES) que sobrevivem com dependência de dispositivos, de intervençôes invasivas, e demandam de serviços especializados e de profissionais qualificados. ${ }^{(7,8)}$

Entre os profissionais de saúde, a equipe de enfermagem é aquela responsável pelo cuidado prolongado e continuo junto a sua clientela. Os conceitos centrais da profissão, sistematizado e científico fundamentados por um referencial teórico facilita a compreensão das experiências humanas e sua interfaces na saúde e na doença. ${ }^{(9)}$

Desta forma, a enfermagem compóe o grupo de categorias profissionais que ensinam e auxiliam os familiares para a adaptaçáo ao cuidado com CRIANES. A família que vivencia a experiência de ter uma criança com necessidades especiais de saúde, precisará se reorganizar e adaptar à novas as rotinas de cuidado. Para além dessas atividades, os familiares precisarão estar disponíveis ao aprendizado da manipulaçáo de dispositivos invasivos que estão associados à manutenção das suas funçóes vitais das crianças, como a ventilação mecânica, dentre outros. $^{(10)}$

Nesse sentido, o Modelo de Adaptação de Calista Roy demonstra-se como uma teoria adequada para nortear este cuidar, pois considera a pessoa como um sistema adaptável, capaz de se ajustar as mudanças no meio ambiente, afetando-o, tendo a enfermagem o papel de promover a adaptação em situaçôes de saúde e doença, aumentando a interação com o ambiente e promovendo saúde. ${ }^{(9)}$

Identificamos lacunas na produção científica relacionados aos fatores que influenciam os cuidados para a desospitalização de crianças em ventilação mecânica e nas açôes de enfermagem para a realização desse cuidado. ${ }^{(8,10)}$ No que tange às crianças em ventilação mecânica, há um déficit de dados disponíveis em países em desenvolvimento, sobre a oferta de serviços que oferecem a ventilação mecânica prolongada, nos domicílios, para pacientes pediátricos. ${ }^{(1)}$ Estudo americano relevou que são limitadas as evidências sobre as repercussôes da qualidade dos cuidados prestados a crianças com necessidades especiais de saúde, quando existe dificuldades sociais e financeiras dos familiares. ${ }^{(12)}$

Assim, conhecer como tem sido as açóes de enfermagem para planejamento da adaptação de familiares para a desospitalização de crianças dependentes de ventilaçáo mecânica poderá auxiliar a minimizar os desafios desse cuidado e potencializar as ações de enfermagem para prática de assistencial qualificada. Diante do exposto, o objetivo desse estudo é apreender as açóes de enfermagem no preparo de familiares para a desospitalização de crianças dependentes de ventilação mecânica. 


\section{Métodos}

Trata-se de um estudo descritivo de abordagem qualitativa, desenvolvido em uma Unidade para desospitalização de um Hospital Pediátrico, localizado em um município de grande porte do estado da Bahia. Essa unidade é referência para o treinamento de cuidadores de crianças em ventilação mecânica que estão em processo de desospitalização. Cerca de 45 crianças são acompanhadas pela unidade que consta com uma unidade de internação e um serviço de Home Care com equipes multidisciplinares formadas por enfermeiros, técnicos de enfermagem, médicos, fonoaudiólogos, nutricionistas, fisioterapeutas, psicólogos e assistentes sociais. ${ }^{(13)}$ Com 28 profissionais de enfermagem e 15 leitos, a unidade de internação está organizada e adaptada para receber familiares e crianças dependentes de ventilação mecânica que passarão pelo processo de adaptação para a desospitalização. Nesse cenário é possível realizar com a família a simulação do cuidado com a criança em ventilação mecânica no domicílio.

Participaram do estudo 15 profissionais, sendo 10 técnicas de enfermagem e cinco enfermeiras. Foram incluídas no estudo somente profissionais da equipe de enfermagem com experiência no processo de desospitalização (compreendido como período da admissão até a alta hospitalar da criança). Os critérios de exclusão foram: profissionais de enfermagem que desempenhavam funçóes temporárias na unidade.

O convite para participação na pesquisa foi realizado durante o turno de trabalho e após o aceite, foi agendado data, local, horário das entrevistas que ocorreram de forma individualizada em local que garantiu privacidade e comodidade das participantes. A coleta de dados foi realizada por meio de entrevista semiestruturada, sendo a primeira parte relativa à caracterização dos participantes (idade, tempo de atuação no serviço, formação profissional, capacitação e cursos de pós-graduação) e a segunda parte com as seguintes questóes norteadoras: quais as açóes que você desenvolve para o preparo do processo de desospitalização de crianças dependentes de ventilação mecânica? Como ocorre o processo de desospitalização dessas crianças?

No período de junho a setembro de 2017 foram realizadas as entrevistas pela própria pesquisadora, em uma sala de reunióes, privativa, no próprio hospital, com duração média de uma hora. As entrevistas foram gravadas em formato de mp3 e transcritas na íntegra. Ao final de cada entrevista houve um feedback com cada participante a fim de garantir as impressóes iniciais dos dados coletados e após o encerramento do estudo a pesquisadora apresentou os resultados as participantes para a validação dos mesmos. A finalização da coleta deu-se pela saturação dos dados, ou seja, quando as respostas durante as entrevistas passaram a ser repetitivas segundo a descrição de diferentes participantes, e portanto, atingindo o objetivo proposto. Essa etapa ocorreu por meio da análise contínua dos dados, desde as entrevistas, das transcriçóes, leituras e discussóes entre as pesquisadoras para a valorização das informaçóes obtidas. Os dados foram submetidos à análise de conteúdo proposta por Bardin, com as fases: organização da análise; codificação; categorização; tratamento dos resultados, inferência e a interpretação dos resultados. ${ }^{(14)}$ Para esse estudo a interpretação dos dados ocorreu à luz dos princípios conceituais do Modelo de Adaptação de Callista Roy (MACR). ${ }^{(15)}$ Nesse modelo teórico, considera-se o indivíduo como como um sistema adaptável pelos seus comportamentos (fisiológico, autoconceito, função da vida real e interdependência) e pelo meio ambiente no qual está inserido, com influência de estímulos focais, contextuais e residuais. ${ }^{(9,16)}$ São estímulos focais, as condiçôes subjetivas, tais como experiências de vida e sentimentos. Contextuais são os elementos ambientais que interferem no indivíduo e contribuem para o efeito do estímulo focal. Os estímulos residuais são fatores, que não são centrais e a pessoa pode ou não ter consciência de sua influência nas experiências vividas. ${ }^{(15,16)}$

O processo de enfermagem segundo MACR constitui uma abordagem para resolução de problemas, por meio da identificação das necessidades das pessoas, implementação de abordagens para o cuidado em enfermagem, bem como a avaliação desse, sendo constituído por seis passos: avaliação do comportamento; avaliação do estímulo; diagnóstico de enfermagem; estabelecimento do objetivo; intervenção e avaliação. ${ }^{(15,16)}$ A partir da análise das falas das profissionais de enfermagem, as açóes realizadas por essa equipe para preparar os familiares formaram as seguintes categorias temáticas seguindo os eixos proposto no modelo teórico de Roy, conforme descrito no quadro 1. 
Quadro 1. Apresentação de recortes das experiências das participantes e formação das categorias temáticas

\begin{tabular}{|c|c|c|}
\hline Experiências compartilhadas & $\begin{array}{l}\text { Eixos do modelo } \\
\text { de Roy }\end{array}$ & $\begin{array}{l}\text { Categorias } \\
\text { temáticas }\end{array}$ \\
\hline $\begin{array}{l}\text { Observamos a mãe... algumas ficam } \\
\text { temerosas... Não sabem o que fazer... como } \\
\text { cuidar. (P03). }\end{array}$ & $\begin{array}{l}\text { Avaliação do } \\
\text { comportamento }\end{array}$ & \multirow[t]{2}{*}{$\begin{array}{l}\text { Reconhecimento dos } \\
\text { problemas }\end{array}$} \\
\hline $\begin{array}{l}\text { Há um preparo da equipe para atender } \\
\text { as demandas da criança e de sua família. } \\
\text { Conhecemos as suas necessidades e a partir } \\
\text { daí iniciamos o treinamento (P01). }\end{array}$ & $\begin{array}{l}\text { Avaliação dos } \\
\text { estímulos da pessoa }\end{array}$ & \\
\hline $\begin{array}{l}\text { A partir das demandas identificadas } \\
\text { pela nossa avaliação é que iniciamos o } \\
\text { treinamento e resolução dos percalços para } \\
\text { desospitalização (P15). }\end{array}$ & $\begin{array}{l}\text { Diagnósticos de } \\
\text { Enfermagem }\end{array}$ & \multirow[t]{2}{*}{$\begin{array}{l}\text { Planejamento do } \\
\text { cuidado para a } \\
\text { desospitalização }\end{array}$} \\
\hline $\begin{array}{l}\text { Nós assumimos o cuidado das crianças até as } \\
\text { mães estarem prontas. Todos os dias fazemos } \\
\text { os procedimentos e pedimos que ela observe, } \\
\text { vamos explicando o passo-a-passo e a } \\
\text { finalidade de cada cuidado. Na medida que se } \\
\text { sentem seguras, elas começam a nos ajudar e } \\
\text { depois já conseguem fazer sozinhas (P08). }\end{array}$ & $\begin{array}{l}\text { Estabelecimento de } \\
\text { objetivos }\end{array}$ & \\
\hline \multirow{2}{*}{$\begin{array}{l}\text { Nós ensinamos a mãe a dar banho no leito..., } \\
\text { manipular o aparelho de ventilação mecânica. } \\
\text { Quando estão prontas, vão para o leito } \\
\text { de simulação para avaliar a segurança do } \\
\text { cuidado (P02). }\end{array}$} & Intervenção & \multirow[t]{2}{*}{$\begin{array}{l}\text { Avaliação do processo } \\
\text { adaptativo para a } \\
\text { desospitalização }\end{array}$} \\
\hline & Avaliação & \\
\hline
\end{tabular}

O estudo foi submetido e aprovado pelo Comitê de Ética em Pesquisa da Escola de Enfermagem da Universidade Federal da Bahia (Parecer consubstanciado: 2.125.417). Foi elaborado um termo de consentimento livre e esclarecido conforme resoluçóes 466/12 e 510/2016 do Conselho Nacional de Saúde. Para garantir o anonimato, as participantes foram identificadas pela letra $\mathrm{P}$, com numeração de 1 a 15 .

\section{Resultados}

Todas as participantes do estudo eram do sexo feminino, com idade entre 25 e 50 anos, com mais de 5 anos de experiência profissional. Entre as dez técnicas de enfermagem, seis referiram ter atualização em pediatria. Dentre as enfermeiras, duas eram especialistas em terapia intensiva.

Considerando que a promoção da adaptação de cuidadores de CRIANES em processo de desospitalização ocorre, por vezes, de forma simultânea, apresentamos as categorias a seguir com a intenção de elucidar as açóes de enfermagem nesse processo: reconhecimento dos problemas; planejamento do cuidado para a desospitalização; avaliação do processo.

\section{Reconhecimento dos problemas}

Entre as ações da enfermagem para promover a adaptação dos cuidadores diante às necessidades das crianças, identificou-se que a equipe buscou realizar levantamento dos estímulos e comportamentos dos cuidadores. Os estímulos focais, nesse estudo foi reconhecido pelas açôes da equipe de enfermagem em valorizar e reconhecer a importância do impacto da doença na vida da família, dos sentimentos, dos anseios e expectativas da família:

Quando as mães chegam aqui elas têm medo de dar banho, têm medo de errar, de cuidar por causa da ventilação mecânica das crianças, a gente mostra diariamente toda a técnica de dar o banho nos meninos, como forrar o leito, como cortar as unhas $e$ depois que elas observam bastante; começam a fazer os cuidados (P05).

Os pais chegam aqui com medo de pegar em seus filhos, de dar banho, de cuidar. Aos poucos vamos juntos vencendo isso, ensinando, e quando eles percebem já sabem até aspirar a traqueostomia (P10).

A equipe realizou o levantamento do estímulo contextual que se revelaram como situaçóes desafiadoras para o planejamento das açóes de enfermagem como as carências econômicas/sociais/educacionais. $O$ analfabetismo ou a baixa escolaridade foram exemplificados pelas participantes:

Fomos ensinar a mãe a administrar os medicamentos e percebemos a dificuldade em entender os nomes e o volume dos medicamentos, mesmo ela dizendo que sabia ler. Conversamos com ela, aos poucos, e ela assumiu que não sabia ler (P13).

Se a mãe tem alguma dificuldade para ler, a gente conversa com ela e organiza o treinamento de outra forma (P01).

As participantes do estudo trouxeram observaçôes que podemos interpretar como 'estímulos residuais', que são outros fatores que influenciam no preparo para a desospitalização, como o cansaço dos familiares. 
O treinamento dos cuidados às crianças é mais fácil quando a familia realmente está empenhada ... e não deixa todos cuidados só para a mãe. Quando tem vários familiares envolvidos no processo de desospitalização...pai, avós, tios... é melhor, não ficam muito cansados, têm a possibilidade de revezamento (P11).

Tem mães que estão nessa situação há anos, sem ir para casa, sem contato com outros familiares e essa rotina cansativa do hospital às vezes prejudica a concentração do treinamento (P03).

Temos que lidar com a máe que, devido estar muito tempo internada, está estressada, cansada e nem sempre está disponivel para aprender (P07).

A incerteza da alta hospitalar, a imprevisibilidade, o quadro instável da criança, foram compartilhadas pelas participantes como fatores que influenciaram no comportamento dos cuidadores e consequentemente no planejamento das açóes da equipe.

Nós percebemos que a mãe ou o pai que não tem alta prevista parecem não se dedicar o suficiente quando comparamos aos que tem uma desospitalização programada, eles mudam... não sei se ficam desestimulados (P04).

Às vezes a família está indo bem no treinamento e o quadro da criança agrava, pela própria doença, indo para UTI. A família desestabiliza, ficando com medo de ir para casa, de cuidar (P06).

As experiências compartilhadas pelas profissionais de enfermagem revelaram que avaliação dos estímulos focal, contextual e residual foram determinantes para a compreensão dos comportamentos adaptativos dos cuidadores e direcionaram o planejamento de suas açóes.

\section{Planejamento do cuidado para a desospitalização} $\mathrm{Na}$ equipe de enfermagem é de responsabilidade da enfermeira realizar o processo e planejamento da assistência. As participantes enfermeiras relataram a realização do levantamento, interpretação dos pro- blemas identificados e a realização do planejamento de suas atividades assistenciais.

A partir das demandas identificadas pela nossa avaliação é que começamos o treinamento e resolução dos desafios para a desospitalização. Realizamos levantamento dos problemas, identificamos as necessidades, planejamos as intervençôes, realizamos as açôes e avaliamos (P15).

As mães entram aqui de uma forma e saem de outra. Preparadas, seguras. Normalmente quando é preciso voltar para o hospital e internar, náo é por falha no cuidado dos familiares ..., mas é pela gravidade da doença (P12).

Apesar das enfermeiras desse estudo não utilizarem uma taxonomia para descrever os diagnósticos, foi possível identificar em seus relatos que realizam a interpretação dos problemas identificados.

As crianças e sua familia veem de outros serviços e o que nós fazemos é avaliar o conhecimento que elas tem, as atividades que desenvolvem e as fragilidades apresentadas. A partir dessa análise é que traçamos nosso plano de cuidado (P04).

Algumas crianças retornavam de suas casas por formar bexigoma. Percebemos que era preciso melhorar o treinamento, reforçando a sondagem vesical para todas as famílias (P14).

Percebe-se que as participantes estão envolvidas no processo de julgamento e interpretação, das necessidades apresentadas pelos familiares e crianças, estabelecendo objetivos e metas para que o cuidador e sua criança consigam retornar ao seu domicílio com as habilidades necessárias para a assistência.

\section{Avaliação do processo adaptativo para a desospitalização}

A avaliação das etapas para o preparo dos cuidadores foi realizada desde o momento da admissáo. As participantes compartilham a experiência dessa ação revelando o compromisso e atenção para com crianças e familiares: 
Primeiro nós observamos como a família cuida, depois vamos realizando o treinamento e avaliando tudo que elas fazem (P09).

Nós avaliamos cada cuidador quando permitimos que ele realize as atividades que ensinamos. Consideramos como apto ou náo apto, à medida que o familiar consegue desempenhar com destreza e segurança o cuidado ao seu filho (P12).

Outro aspecto na fase de avaliação realizado pela enfermagem, foi a existência de um leito de simulação da realidade domiciliar, na unidade, onde a família é acompanhada, recriando um ambiente similar ao domicílio, incluindo também os equipamentos, para que o cuidador seja avaliado quanto a segurança e destreza nos cuidados.

Para a família entrar na simulação precisa estar preparada e apresentar segurança no cuidado à criança (P02).

Depois que ensinamos..., e que as condiçóes sociais e financeiras estão favoráveis a alta hospitalar, a criança entra em simulação. O cuidado passa a ser do pai ou da máe. Se ele nos requisitar ou demonstrar muitas duividas, a criança volta novamente ao treinamento (P08).

\section{Discussão}

Os dados analisados revelaram que as açóes de enfermagem para a alta das crianças em ventilação mecânica têm como objetivo a promoção de um cuidado adaptativo. Visto que contemplam desde o preparo técnico para que os cuidadores aprendam a manipular equipamentos, realizar cuidados específicos com suporte ventilatório e manutenção das vias aéreas, assim como preparam os cuidadores com respeito aos estímulos focais, contextuais e residuais que permeiam as experiências dos familiares.

Nesse estudo, percebe-se a realização das etapas propostas pelo modelo teórico de Roy, pela atenção $\mathrm{da}$ equipe em valorizar as demandas dos familiares para o planejamento da assistência, corroborando com os achados de estudo com adolescentes asmáti- cos sob a ótica de Roy, realizado no Irã, desvelando que o envolvimento da família possui contribuiçôes importantes para o fortalecimento do processo adaptativo, com redução no impacto da doença. ${ }^{(17)}$

Aspectos sociais, econômicos e baixa escolaridade, estímulos contextuais, interferem no processo de adaptação e preparo para a alta. Tais fatores necessitam de relação dialógica que acolha as duvidas e demandas da família para um planejamento efetivo e de qualidade na prestação dos cuidados. ${ }^{(18,19)} \mathrm{A}$ carência de rede social que permeiam o cotidiano das crianças conduz ao desgaste de seus pais, principalmente a mãe, que é considerada na maioria dos estudos a principal cuidadora da criança com necessidade especial. ${ }^{(8)}$ A valorização dos saberes relacionais que fundamentam as necessidades afetivas e sociais dos cuidadores deve estar presente nas açóes de promoção de adaptação pela equipe de enfermagem, como apoio e fortalecimento e preservação da saúde física e mental dos cuidadores. ${ }^{(20-22)}$

Como parte integrante do processo de enfermagem, os diagnósticos foram realizados de modo assistemático sem um método ou uso de taxonomia. Fator que pode fragilizar as avaliaçóes dos resultados da assistência. $\mathrm{O}$ uso de uma terminologia padronizada na identificação e representação do diagnóstico de enfermagem favorece a comunicação entre enfermeiros e outros membros da equipe, melhora a continuidade do cuidado, favorece o registro, documentação, e torna padronizado as açôes de enfermagem. ${ }^{(23)}$

As atividades de intervenção realizadas pela equipe baseou-se na observação, realizaçáo de cuidados em parceria com os familiares e por meio da avaliação contínua assumindo o papel de educador e mediador de aprendizado, cujo processo de ensino centraliza-se no cuidado procedimental e na mediaçáo de saberes. ${ }^{(8)}$

O planejamento e a avaliação dos resultados das intervençôes de enfermagem foram direcionados para corrigir as possíveis falhas e fragilidades dos familiares cuidadores. Não foi revelado atitudes das profissionais que reforçavam as açóes positivas realizadas pelos cuidadores, contraponto o que a teoria preconiza que os comportamentos adaptáveis sejam valorizados e aumentados para fortalecer e promover atitudes assertivas. ${ }^{(15)}$ Embora as participantes do estudo não façam referência às etapas do processo de 
enfermagem segundo o MACR, percebe-se os princípios da fundamentação teórica na prática assistencial.

\section{Conclusão}

As ações de enfermagem aconteceram por meio do preparo dos familiares das crianças dependentes de ventilação mecânica e foram desveladas pelo levantamento e reconhecimento dos problemas, pelo planejamento do cuidado e na avaliação do processo de adaptação para alta. As açóes desenvolvidas pelas participantes do estudo consideraram o indivíduo de forma adaptativa compreendendo os diversos estímulos e interferências que afetam as crianças e suas famílias. A compreensão das necessidades evidenciadas na transição do hospital para o domicílio poderá proporcionar autonomia e segurança à família. As limitaçóes desse estudo dizem respeito, ao cenário, o que reflete a imagem apenas do universo estudado. Além disso, não foi explorado as percepçóes dos familiares cuidadores sobre sua adaptação para a desospitalização, o que direcionou a discussão em apenas uma perspectiva. $\mathrm{O}$ uso de uma teoria de enfermagem fortalece as açóes na prática clínica e assistencial.

\section{Colaborações}

Ulisses LO, Bispo TAS, Caldas AB, Camargo CL, Oliveira MMC, Silva EA, Gomes NP e Whitaker MCO contribuíram com a concepção do projeto, redação do artigo, revisão crítica relevante do conteúdo intelectual, análise e interpretação dos dados e aprovação final da versão a ser publicada.

\section{Referências}

1. Amin R, MacLusky I, Zielinski D, Adderley R, Carnevale F, Chiang J, et al. Pediatric home mechanical ventilation: a Canadian Thoracic Society clinical practice guideline executive summary. Canadian J Respiratory, Critical Care, Sleep Med. 2017;1:1,7-36.

2. Edwards JD, Morris MC, Nelson JE, Panitch HB, Miller RL. Decisions around Long-term Ventilation for Children. Perspectives of Directors of Pediatric Home Ventilation Programs. Ann Am Thorac Soc. 2017;14(10):1539-47.

3. Center for Disease Control and Prevention (CDC). Caring for Children in a Disaster. Children and youth with special healthcare needs in emergencies. United States: CDC; 2019 [cited 2020 Jan 28]. Available from: https://www.cdc.gov/childrenindisasters/children-with-specialhealthcare-needs.html

4. Falkson S, Knecht C, Hellmers C, Metzing S. The Perspective of families with a ventilator-dependent child at home. A literature review. J Pediatr Nurs. 2017;36:213-224. Review.

5. São Paulo. Secretaria de Direitos Humanos da Presidência da República. Características da População Brasileira. Brasília (DF): Secretaria Nacional de Promoção dos Direitos da Pessoa com Deficiência Censo 2010 [citado 2020 Jan 20]. Disponível em: http:// www.pessoacomdeficiencia.gov.br

6. Moreira MC,Albernaz LV, Sá MR, Correia RF, Tanabe RF. Recomendações para uma linha de cuidados para crianças e adolescentes com condições crônicas complexas de saúde. Cad Saude Publica. 2017;33(11):e00189516.

7. Estrem B, Wall J, Paitich L, Maynard R. The ventilator-dependent child: what every home care nurse needs to know. Home Healthc Now. 2020;38(2):66-74. Review.

8. Goes FG, Cabral IE. Discourses on discharge care for children with special healthcare needs. Rev Bras Enferm. 2017;70(1):154-61.

9. Jennings KM. The roy adaptation model: a theoretical framework for nurses providing care to individuals with anorexia nervosa. ANS Adv Nurs Sci. 2017;40(4):370-83.

10. Góes FG, Cabral IE. Hospital discharge in children with special health care needs and its different dimensions. Rev Enferm UERJ. 2017;25:e18684.

11. Hanashiro M, Franco AO, Ferraro AA, Troster EJ. Care alternatives for pediatric chronic mechanical ventilation. J Pediatr (Rio J). 2011;87(2):145-9.

12. Kan K, Choi H, Davis M. Immigrant families, children with special health care needs, and the medical home. Pediatrics. 2016;137(1).

13. Hospital Martagão Gesteira. Home care: hospital Martagão Gesteira amplia assistência domiciliar. Savador (BA): Hospital da Criança; 2019 [citado 2020 Jan 20]. Disponível em: http://martagaogesteira.com.br/home-care/

14. Bardin L. Análise de conteúdo. Lisboa: Edições 70; 2011. 281 p.

15. Roy C. Extending the Roy adaptation model to meet changing global needs. Nurs Sci Q. 2011;24(4):345-51.

16. Diaz LJ, Cruz DA. Adaptation model in a controlled clinical trial involving family caregivers of chronic patients. Texto Contexto Enferm. 2017;26(4):e0970017.

17. Alimohammadi N, Maleki B, Abbasi S, Shakerian B, Hemati Z. The effect of adaptation training on controlling maladaptation behaviors in adolescents with asthma based on roy adaptation model. Tanaffos. 2018;17(2):103-9.

18. Dias BC, Arruda GO, Marcon SS. Vulnerabilidade familiar de crianças com necessidades especiais de cuidados múltiplos, complexos e contínuos. Rev Min Enferm. 2017;21:e1027.

19. Amador DD, Marcílio AC, Soares JS, Marques FR, Duarte AM, Mandetta MA. The strength of information on retinoblastoma for the family of the child. Acta Paul Enferm. 2018;31(1):87-94.

20. Figueiredo SV, Sousa AC, Gomes IL. Children with special health needs and family: implications for Nursing. Rev Bras Enferm. 2016;69(1):79-85.

21. Fernandes CS, Angelo M. Family caregivers: what do they need? An integrative review. Rev Esc Enferm USP. 2016;50(4):672-8.

22. Cruz CT, Zamberlan KC, Silveira A, Buboltz FL, Silva JH, Neves ET. Atenção à criança com necessidades especiais de cuidados contínuos e complexos: percepção da enfermagem. Rev Min Enferm. 2017;21:e-1005.

23. Felix ND, Ramos NM, Nascimento MN, Moreira TM, Oliveira CJ. Nursing diagnoses from ICNPR for people with metabolic syndrome. Rev Bras Enferm. 2018;71(Suppl 1):467-74. 